\title{
Assessment of agreement and clinical interchangeability between the TEG5000 and TEG6S thromboelastography haemostasis analysers: a prospective validation study
}

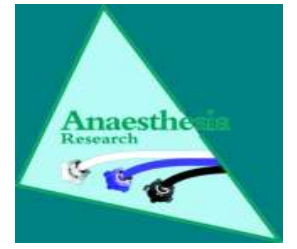

BACKGROUND:Thromboelastography (TEG) allows rapid, comprehensive and accurate identification of haemostatic condition. Automation provided by the TEG6S $\otimes$ (Haemonetics, Illinois, USA) platform simplifies this process, enabling multiple assays to be performed simultaneously. This is achieved by measuring clot viscoelasticity using resonance technology in a dynamic manner throughout the coagulation process. The aim of this study was to assess agreement and clinical interchangeability between TEG6S and TEG $₫ 5000$ analysers.

METHODS: After Ethics committee approval, we performed a prospective validation study assessing interdevice agreement between TEG6S and TEG5000 analysers. Whole blood $(3.5 \mathrm{~mL})$ was sampled in citrated tubes from 25 adult patients in tertiary level intensive care unit (ICU). Each sample was analysed via two TEG6S systems (Machine 1 \& Machine 2) and a single TEG5000 system, simultaneously. A trained operator performed all the measurements on each device. We used Lin's concordance and Intraclass correlation coefficients to assess agreement, and Reduced Major Axis regression to examine fixed and proportional components of disagreement.

RESULTS: Sixteen (64\%) patients were male; the mean (SD) age was 61 (17) years. Admission ICU diagnosis: post-cardiac surgery $(24 \%)$, decompensated liver disease (16\%), post-liver transplantation (16\%), post-general surgery $(8 \%)$, neurological injury $(16 \%)$, other critical illness $(12 \%)$. We found that the TEG6S and TEG5000 systems were broadly interchangeable. The majority of TEG variables demonstrated almost perfect or substantial agreement, with minimal fixed bias or proportional bias. Exceptions were MA, which demonstrated a fixed, non-proportional bias and $\mathrm{LY} 30 \%$, which demonstrated proportional bias between TEG6S and TEG5000 analysers. These trends between TEG6S and TEG5000 were consistent across both TEG6S machines used (Tables 1 \& 2), (Figures 1 - 5).

CONCLUSION: In adult patients in the intensive care and post-operative environments, across a variety of pathophysiological states, there was almost perfect agreement in the $R$ time and MA, substantial agreement in K-time and alpha angle, and poor LY30\% agreement between the TEG6S and TEG5000 analysers. The results of this study indicate that TEG6S and TEG5000 platforms are clinically interchangeable, demonstrating no substantial proportional bias, with the exception of clot lysis. This has important implications for use in clinical practice and in multi-site research programs.
Donald
Prof. Leonid Churilov ${ }^{3}$, A/Prof. Laurence Weinberg

of Anaesthesia ${ }^{1}$ \& Intensive Care ${ }^{2}$, Austin Hospital, Victoria, Australia

Correspondence: laurence.weinberg@austin.org.au
7.F. THE UNIVERSITY OF 65. $\overline{\text { MELBOURNE }}$

A Austin Health

Table 1. Machine 1 (TEG6S) and TEG5000 agreement

\begin{tabular}{|l|l|l|l|l|}
\multicolumn{1}{|c|}{ Parameter } & \multicolumn{1}{|c|}{$\begin{array}{c}\text { Lin's concordance coeff. } \\
(\mathbf{9 5 \%} \mathbf{C l})\end{array}$} & \multicolumn{1}{|c|}{ Intercept } & \multicolumn{1}{|c|}{$\begin{array}{c}\text { Slope } \\
\text { Intraclass } \\
\text { correlation coeff. }\end{array}$} \\
\hline R time & $0.78(0.64$ to 0.92$)$ & 0.92 & 0.76 & 0.79 \\
\hline K time & $0.82(0.69$ to 0.94$)$ & -0.93 & 1.30 & 0.82 \\
\hline Alpha angle & $0.80(0.64$ to 0.95$)$ & -1.43 & 1.04 & 0.80 \\
\hline Max. Amplitude & $0.90(0.83$ to 0.96$)$ & -5.00 & 1.00 & 0.90 \\
\hline Lysis 30 & $0.34(0.10$ to 0.56$)$ & 0.04 & 0.43 & 0.33 \\
\hline
\end{tabular}

Table 2. Machine 2 (TEG6S) and TEG5000 agreement

\begin{tabular}{|l|l|l|l|l|}
\multicolumn{1}{|c|}{ Parameter } & \multicolumn{1}{|c|}{$\begin{array}{c}\text { Lin's concordance coeff. } \\
(\mathbf{9 5 \%} \mathbf{C l})\end{array}$} & \multicolumn{1}{|c|}{ Intercept } & \multicolumn{1}{|c|}{$\begin{array}{c}\text { Slope } \\
\text { Intraclass } \\
\text { correlation coeff. }\end{array}$} \\
\hline R time & $0.86(0.76$ to 0.96$)$ & 0.41 & 0.86 & 0.87 \\
\hline K time & $0.79(0.65$ to 0.94$)$ & -0.87 & 1.21 & 0.80 \\
\hline Alpha angle & $0.70(0.49$ to 0.91$)$ & -1.74 & 0.86 & 0.71 \\
\hline Max. Amplitude & $0.90(0.84$ to 0.97$)$ & -3.51 & 0.97 & 0.90 \\
\hline Lysis 30 & $0.32(0.12$ to 0.52$)$ & -0.02 & 0.36 & 0.31
\end{tabular}

Figure 1. Agreement between TEG6S and TEG5000 for R-time

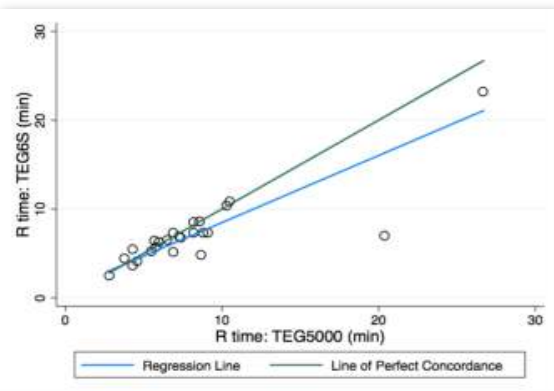

Figure 3. Agreement between TEG6S and TEG5000 for Alpha Angle

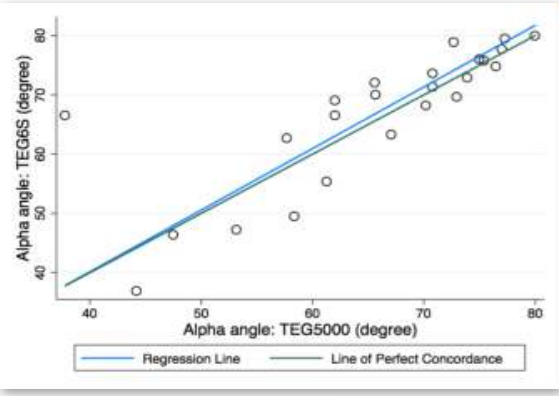

Figure 2. Agreement between TEG6S and TEG5000 for K-time

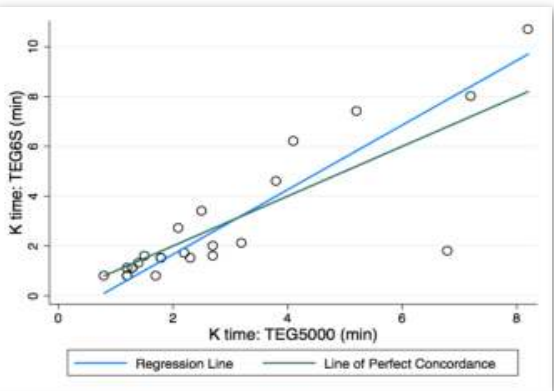

Figure 4. Agreement between TEG6S and TEG5000 for Maximum Amplitude

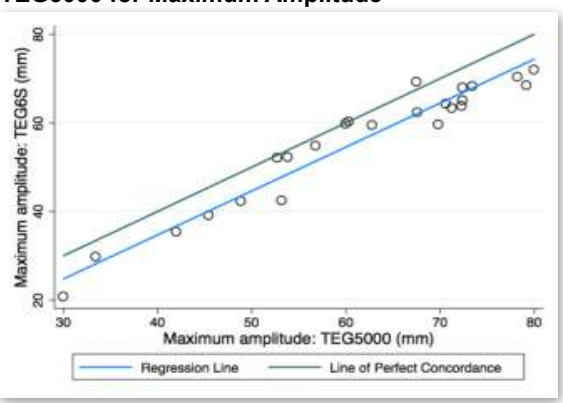

Figure 5.

Agreement between TEG6S and TEG5000 for Lysis (LY30\%)

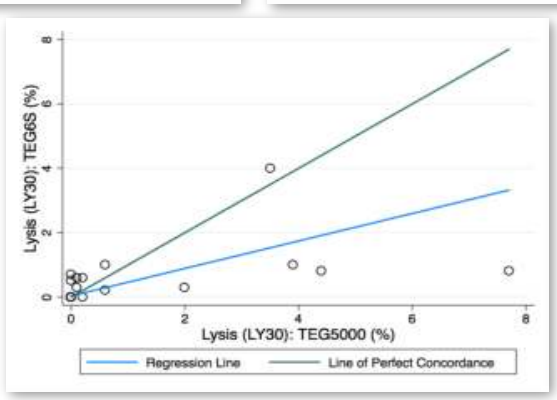

